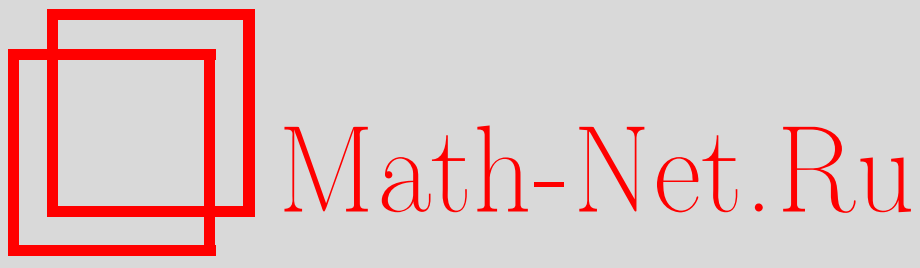

В. А. Копытцев, Предельные теоремы для числа решений системы случайных уравнений, Теория вероятн. и ее примен., 2000, том 45, выпуск 1, 52-72

DOI: https://doi.org/10.4213/tvp324

Использование Общероссийского математического портала Math-Net.Ru подразумевает, что вы прочитали и согласны с пользовательским соглашением http://www.mathnet.ru/rus/agreement

Параметры загрузки:

IP : 54.162 .85 .209

26 апреля 2023 г., 12:58:54

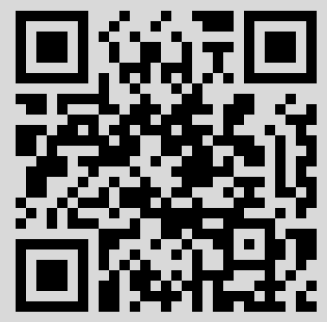


(c) $2000 \mathrm{r}$.

КОПЫТЦЕВ В. А.*

\section{ПРЕДЕЛЬНЫЕ ТЕОРЕМЫ ДЛЯ ЧИСЛА РЕШЕНИЙ СИСТЕМЫ СЛУЧАЙНЫХ УРАВНЕНИЙ}

В статье исследуются число и структура множества решений заведомо совместной системы случайных уравнений вида

$$
\varphi_{t}\left(x_{s_{1}(t)}, \ldots, x_{s_{d(t)}(t)}\right)=a_{t}, \quad t=1, \ldots, T,
$$

относительно переменных $x_{1}, \ldots, x_{n} \in\{0, \ldots, q-1\}, q \geqslant 2$, где индексы $s_{1}(t), \ldots, s_{d(t)}(t)$ выбираются случайно и независимо при разных $t$ посредством процедуры равновероятного выбора без возврашения. Найдены условия, при которых распределение числа решений этой системы уравнений сходится к распределению случайной величины вида $A \cdot 2^{\eta_{1}} \cdots q^{\eta_{q}-1}$, где $A$ - порядок группы подстановок $g:\{0, \ldots, q-1\} \longleftrightarrow\{0, \ldots, q-1\}$, удовлетворяюших условиям $\varphi_{t}\left(y_{1}, \ldots, y_{d(t)}\right) \equiv \varphi_{t}\left(g\left(y_{1}\right), \ldots, g\left(y_{d(t)}\right)\right), t=1, \ldots, T$, а $\eta_{1}, \ldots, \eta_{q-1}$ - независимые случайные величины, распределенные по закону Пуассона с параметрами $\lambda_{1}, \ldots, \lambda_{q-1}$ соответственно. Приведены выражения для параметров $\lambda_{1}, \ldots, \lambda_{q-1}$. Эти результаты сушественно обобщают аналогичные теоремы для случая $q=2$, доказанные в работах [2] и [3].

Ключевые слова и фразы: системы случайных уравнений, истинное решение, охрестность истинного решения, общее число решений, группы подстановок, распределение Пуассона.

\section{1. Введение и формулировка основных результатов}

Пусть $\varphi(y)=\varphi\left(y_{1}, \ldots, y_{d}\right)$ - некоторая функция, заданная на множестве $d$-мерных векторов с координатами $y_{1}, \ldots, y_{d} \in\{0, \ldots, q-1\}$, $q \geqslant 2$. Выбор области значений функции не будет играть никакой роли и не скажется на результатах.

Условимся обозначать через $\Sigma_{\varphi}$ группу инерции функции $\varphi$ в симметрической группе $S_{d}$, определяемую условием

$$
\sigma \in \Sigma_{\varphi} \Longleftrightarrow \varphi\left(y_{\sigma(1)}, \ldots, y_{\sigma(d)}\right) \equiv \varphi\left(y_{1}, \ldots, y_{d}\right)
$$

Рассмотрим случайное уравнение

$$
\varphi\left(x_{s_{1}}, \ldots, x_{s_{d}}\right)=a
$$

${ }^{*}$ ФАПСИ, Б. Кисельный пер., 4, 103031 Москва, Россия. 
относительно переменных $x_{1}, \ldots, x_{n} \in\{0, \ldots, q-1\}$; где индексы $s_{1}, \ldots, s_{d}$ образуют случайную выборку без возврашения из множества $\{1, \ldots, n\}$. Предполагается, что упорядоченные выборки $\left(s_{\sigma(1)}, \ldots, s_{\sigma(d)}\right)$, $\boldsymbol{\sigma} \in \boldsymbol{\Sigma}_{\varphi}$, не различаются и объединяются в один исход. На множестве $\Omega=\{\omega\}$ возможных исходов (классов эквивалентных левых частей уравнения) задано равномерное распределение вероятностей

$$
P_{\varphi}(\omega)=\left[\left(\begin{array}{l}
n \\
d
\end{array}\right) \frac{d !}{\left|\Sigma_{\varphi}\right|}\right]^{-1}, \quad \forall \omega \in \Omega
$$

Пусть система уравнений

$$
\varphi\left(x_{s_{1}(t)}, \ldots, x_{s_{d}(t)}\right)=a_{t}, \quad t=1, \ldots, T
$$

составлена из независимо выбранных уравнений вида (1). Правая часть системы определяется равенствами

$$
a_{t}=\varphi\left(x_{s_{1}(t)}^{\circ}, \ldots, x_{s_{d}(t)}^{\circ}\right), \quad t=1, \ldots, T
$$

при некотором заданном векторе $x^{\circ}=\left(x_{1}^{o}, \ldots, x_{n}^{\circ}\right)$. Вектор $x^{\circ}$ назовем истинным решением.

Предположим теперь, что имеется конечный набор функций

$$
\Phi=\left\{\varphi_{1}, \varphi_{2}, \ldots, \varphi_{R}\right\}
$$

где $\varphi_{r}=\varphi_{r}\left(y_{1}, \ldots, y_{d(r)}\right), y_{1}, \ldots, y_{d(r)} \in\{0, \ldots, q-1\}, r=1, \ldots, R$. Рассмотрим систему уравнений

$$
\begin{gathered}
\varphi_{r}\left(x_{s_{1}(t)}, \ldots, x_{s_{d(r)}(t)}\right)=\varphi_{r}\left(x_{s_{1}(t)}^{\circ}, \ldots, x_{s_{d(r)}(t)}^{\circ}\right) \\
t=1, \ldots, T(r), r=1, \ldots, R
\end{gathered}
$$

составленную из независимо выбранных подсистем вида (3), соответствующих функциям $\varphi_{1}, \ldots, \varphi_{R}$.

Система уравнений (4) является заведомо совместной системой случайных уравнений, имеющей, по крайней мере, решение $x^{\circ}$. Общее число решений $\xi$ является случайной величиной.

Общее число решений $\xi$ для различных классов систем уравнений вида (3) и (4) с функциями $\varphi$, заданными на двоичных векторах, исследовалось в работах [1]-[3]. Новизна данной работы состоит в том, что функции $\varphi$ определены на векторах с координатами из произвольного конечного множества. Отметим, что заведомо совместные системы уравнений исследовались также в [4] и некоторых других работах, но там использована другая схема формирования левых частей.

Приведем формулировки основньх результатов. 
Пусть $i, j \in\{0, \ldots, q-1\}, i \neq j$, и пусть $d_{0}, \ldots, d_{q-1}$ - набор цельхх неотрицательных чисел таких, что $d_{0}+\cdots+d_{q-1}=d(r)-1$. Для каждой Функции $\varphi_{r}=\varphi_{r}\left(y_{1}, \ldots, y_{d(r)}\right) \in \Phi$ положим

$$
\pi_{r}\left(i, j ; d_{0}, \ldots, d_{q-1}\right)=\sum_{k=1}^{d(r)} \pi_{r}^{(k)}\left(i, j ; d_{0}, \ldots, d_{q-1}\right)
$$

где величина $\pi_{r}^{(k)}\left(i, j ; d_{0}, \ldots, d_{q-1}\right)$ равна числу пар $\left(y^{\prime}, y^{\prime \prime}\right)$ векторов вида

$$
\begin{aligned}
y^{\prime} & =\left(y_{1}, \ldots, y_{k-1}, y_{k}^{\prime}, y_{k+1}, \ldots, y_{d(r)}\right) \\
y^{\prime \prime} & =\left(y_{1}, \ldots, y_{k-1}, y_{k}^{\prime \prime}, y_{k+1}, \ldots, y_{d(r)}\right)
\end{aligned}
$$

удовлетворяюших условиям: 1) $y_{k}^{\prime}=i, y_{k}^{\prime \prime}=j$;2) среди координат $y_{\nu}, \nu \neq$ $k$, содержится $d_{w}$ координат, равных $\left.w=0, \ldots, q-1 ; 3\right) \varphi_{r}\left(y^{\prime}\right)=\varphi_{r}\left(y^{\prime \prime}\right)$.

Будем различать два случая:

(A) Для некоторой функции $\varphi_{r} \in \Phi$ найдутся значения $i, j, i \neq j$, и значения $d_{0}, \ldots, d_{q-1}$ такие, что $\pi_{r}\left(i, j ; d_{0}, \ldots, d_{q-1}\right)>0$.

(Б) Для любых $\varphi_{r} \in \Phi, i, j(i \neq j), d_{0}, \ldots, d_{q-1}$ выполняется равенство $\pi_{r}\left(i, j ; d_{0}, \ldots, d_{q-1}\right)=0$.

Зафиксируем набор чисел

$$
0<\theta_{i}<1, \quad \sum_{i=0}^{q-1} \theta_{i}=1,
$$

и введем величины

$$
\begin{gathered}
\lambda_{r}(i, j)=\sum_{\substack{d_{0}, \ldots, d_{q-1} \\
d_{0}+\cdots+d_{q-1}=d(r)-1 \\
\lambda_{r}^{*}=\max _{i, j} \lambda_{r}(i, j) .}} \theta_{0}^{d_{0}} \cdots \theta_{q-1}^{d_{q-1}} \pi_{r}\left(i, j ; d_{0}, \ldots, d_{q-1}\right), \\
\end{gathered}
$$

Пусть выполняется случай (А). Сопоставим функциям $\varphi_{r}: \lambda_{r}^{*}>0$ некоторые фиксированные числа $t(r)>0$. Немного ниже мы определим связь этих чисел с параметрами $T(r)$ и $n$. Положим

$$
\begin{gathered}
\lambda(i, j)=\sum_{r: \lambda_{r}^{*}>0} t(r) \lambda_{r}(i, j), \quad \lambda^{*}=\max _{i, j} \lambda(i, j), \\
M=\left\{(i, j): \lambda(i, j)=\lambda^{*}\right\} .
\end{gathered}
$$

Отметим, что, согласно определению, $\lambda^{*}>0$.

Каждому элементу $i \in\{0, \ldots, q-1\}$ поставим в соответствие множество

$$
J_{i}=\{j:(i, j) \in M\}
$$


Всякое непустое множество (10) разобьем на классы эквивалентных элементов

$$
J_{i}=J_{1}^{(1)}+\cdots+J_{i}^{\left(N_{i}\right)}, \quad N_{i} \geqslant 1,
$$

с учетом отношения эквивалентности: $j_{1} \sim j_{2}$, если для любой функции $\varphi_{r}: \lambda_{r}^{*}>0$ каждое равенство $\varphi_{r}\left(y^{\prime}\right)=\varphi_{r}\left(y^{\prime \prime}\right)$, которое выполняется при некоторых векторах $y^{\prime}, y^{\prime \prime}$ вида (6), где $y_{k}^{\prime}=i$, $y_{k}^{\prime \prime}=j_{1}, k \in\{1, \ldots, d(r)\}$, влечет равенство $\varphi_{r}\left(y^{\prime}\right)=\varphi_{r}\left(y^{\prime \prime \prime}\right)$, где $y^{\prime \prime \prime}=$ $\left(y_{1}, \ldots, y_{k-1}, y_{k}^{\prime \prime \prime}, y_{k+1}, \ldots, y_{d(r)}\right), y_{k}^{\prime \prime \prime}=j_{2}$.

Для описания свойств числа $\xi$ и множества $\Xi$ решений системы уравнений (4) нам понадобятся также следующие определения.

Пусть $g^{\prime}:\{0, \ldots, q-1\} \longrightarrow\{0, \ldots, q-1\}$ - некоторое отображение множества $\{0, \ldots, q-1\}$ в себя, и пусть $y=\left(y_{1}, \ldots, y_{d(r)}\right)-$ вектор с координатами из этого множества. С целью упрощения обозначений будем полагать $g^{\prime}(y)=\left(g^{\prime}\left(y_{1}\right), \ldots, g^{\prime}\left(y_{d(r)}\right)\right)$.

Обозначим $G_{r}$ группу подстановок $g:\{0, \ldots, q-1\} \longleftrightarrow\{0, \ldots, q-1\}$, удовлетворяющих условию $\varphi_{r}(y) \equiv \varphi_{r}(g(y))$, и положим

$$
G=\bigcap_{r=1}^{R} G_{r}
$$

Ясно, что если некоторый вектор $x=\left(x_{1}, \ldots, x_{n}\right)$ является решением системы (4), то ее решением является любой вектор $g(x)=$ $\left(g\left(x_{1}\right), \ldots, g\left(x_{n}\right)\right), g \in G$. Следовательно, любой вектор $g\left(x^{\circ}\right), g \in G$, является решением этой системы.

Зафиксируем некоторую подстановку $g \in G$ и сопоставим множествам

$$
g J_{i}^{(l)}=\left\{g(j): j \in J_{i}^{(l)}\right\}, \quad i: J_{i} \neq \varnothing, l=1, \ldots, N_{i}
$$

множества индексов

$$
S_{t, r}\left(g J_{i}^{(l)}\right) \subseteq\left\{s: g\left(x_{s}^{\circ}\right)=g(i)\right\}=\left\{s: x_{s}^{\circ}=i\right\}=\{s(i)\}
$$

которые определим условием: $s(i) \in S_{t, r}\left(g J_{i}^{(l)}\right) \Longleftrightarrow$ любой вектор $\left(x_{1}, \ldots, x_{n}\right) \in X_{t, r}$, где

$$
\begin{gathered}
x_{s}=g\left(x_{s}^{\circ}\right), \quad s \neq s(i) \\
x_{s(i)} \in g(i) \cup g J_{i}^{(l)} \quad\left(g(i)=g\left(x_{s(i)}^{\circ}\right)\right)
\end{gathered}
$$

$X_{t, r}$ - множество решений уравнения с номером $t \in\{1, \ldots, T(r)\}$ в подсистеме уравнений с функцией $\varphi_{r}$.

Отметим, что, согласно этому определению,

$$
S_{t, r}\left(g J_{i}^{(l)}\right)=S_{t, r}\left(J_{i}^{(l)}\right), \quad \forall g \in G .
$$


Положим

$$
S_{i}^{(l)}=\bigcap_{r=1}^{R} \bigcap_{t=1}^{T(r)} S_{t, r}\left(g J_{i}^{(l)}\right)
$$

Каждое переменное $x_{s(i)}$ с индексом $s(i) \in S_{i}^{(l)}$ можно называть несущественным относительно решения $g\left(x^{\circ}\right)=\left(g\left(x_{1}^{\circ}\right), \ldots, g\left(x_{n}^{\circ}\right)\right)$ по множеству значений $g(i) \cup g J_{i}^{(l)}$. Это название мотивируется тем, что, по определению множеств $S_{i}^{(l)}$, решениями системы (4), кроме решения $g\left(x^{\circ}\right)$ (где $\left.x_{s(i)}=g\left(x_{s(i)}^{\text {o }}\right)=g(i)\right)$, являются все векторы, отличные от вектора $g\left(x^{\circ}\right)$ только по значениям переменной $x_{s(i)}$ и в которых переменная $x_{s(i)}$ может принимать любое значение из множества $g J_{i}^{(l)}$.

Относительно системы $\left\{S_{i}^{(l)}\right\}$ подмножеств $S_{i}^{(l)}$, где $i: J_{i} \neq \varnothing$, $l=1, \ldots, N_{i}$, определим множество векторов вида

$$
\begin{aligned}
X_{g\left(x^{\circ}\right)}\left(\left\{S_{i}^{(l)}\right\}\right)=\{ & \left(x_{1}, \ldots, x_{n}\right): x_{s}=g\left(x_{s}^{\circ}\right), \forall s \notin S_{i}^{(l)} ; \\
& \left.x_{s} \in g(i) \cup g J_{i}^{(l)}\left(g(i)=g\left(x_{s}^{\circ}\right)\right) \forall s \in S_{i}^{(l)}\right\} .
\end{aligned}
$$

Это множество содержит вектор $g\left(x^{\circ}\right)$ и все векторы, отличные от $g\left(x^{\circ}\right)$ по значениям переменных с индексами из множеств $S_{i}^{(l)}$ : для этих переменных допускаются все значения из множеств $g(i) \cup g J_{i}^{(l)}$. Таким образом,

$$
\left|X_{g\left(x^{\circ}\right)}\left(\left\{S_{i}^{(l)}\right\}\right)\right|=\prod_{i: J_{i} \neq \varnothing} \prod_{l=1}^{N_{i}}\left|g(i) \cup g J_{i}^{(l)}\right|^{m_{i}^{(l)}}=\prod_{i: J_{i} \neq \varnothing} \prod_{l=1}^{N_{i}}\left(1+h_{i}^{(l)}\right)^{m_{i}^{(l)}},
$$

где $m_{i}^{(l)}=\left|S_{i}^{(l)}\right|, h_{i}^{(l)}=\left|g J_{i}^{(l)}\right|=\left|J_{i}^{(l)}\right|, \forall g \in G$.

Теорема 1. Пусть выполняется случай (A) $u$, кроме того,

1) все функиии $\varphi_{r} \in \Phi=\left\{\varphi_{1}, \ldots, \varphi_{R}\right\}$ обладают свойством: $\partial л я$ любых элементов $i, j \in\{0, \ldots, q-1\}, i \neq j$, найдется пара векторов $y^{\prime}, y^{\prime \prime}$ вида (6), где $y_{k}^{\prime}=i, y_{k}^{\prime \prime}=j, k \in\{1, \ldots, d(r)\}$, и таких, что $\varphi_{r}\left(y^{\prime}\right) \neq \varphi_{r}\left(y^{\prime \prime}\right)$

npu $n \rightarrow \infty$

2) истинное решение содержит $n_{i}$ координат, принимающих значение $i$, причем $n_{i}=\theta_{i}(n) \cdot n, \theta_{i}(n)=\theta_{i}+o\left(\ln ^{-1} n\right), 0<\theta_{i}<1$;

3) величины $T(r)$ связаны с иислом неизвестных $n$ соотношениями 3.1) $T(r) / n \rightarrow \infty, r=1, \ldots, R$,

3.2) $T(r)=T \cdot t(r)+o(n) \forall r: \lambda_{r}^{*}>0$,

3.3) $\sum_{r=1}^{R} T(r) d(r)-T \lambda^{*}=n(\ln n+z), z=O(1)$.

Tогда прu $n \rightarrow \infty$

1) предельное распределение числа решений $\xi$ имеет вид

$$
\mathbf{P}\{\xi=w\}=\sum \prod_{i: J_{i} \neq \varnothing} \prod_{l=1}^{N_{i}} \frac{\left(\theta_{i} e^{-z}\right)^{m_{i}^{(l)}}}{m_{i}^{(l)} !} \exp \left\{-\theta_{i} e^{-z}+o(1)\right\},
$$


сумма $\sum$ берется по значениям величин $m_{i}^{(l)}, i: J_{i} \neq \varnothing, l=\overline{1, N_{i}}$, таким, umo

$$
w=|G| \prod_{i: J_{i} \neq \varnothing} \prod_{l=1}^{N_{i}}\left(1+h_{i}^{(l)}\right)^{m_{i}^{(l)}}
$$

где $|G|$ - порядок группы подстановок (12);

2) структура множества $\Xi$ решений описывается предельным соотношением

$$
\mathbf{P}\left\{\Xi=\bigcup_{g \in G} X_{g\left(x^{0}\right)}\left(\left\{S_{i}^{(l)}\right\}\right)\right\} \longrightarrow 1
$$

Следствие. В условиях теоремы 1 распределение числа решений слабо сходится $x$ распределению случайной величины $|G| \cdot 2^{\eta_{1}} \cdots q^{\eta_{q-1}}$, где $\eta_{1}, \ldots, \eta_{q-1}$ - независимые случайные величины, распределенные по закону Пуассона с параметрами $\lambda_{1}, \ldots, \lambda_{q-1}$ соответственно,

$$
\lambda_{h}=e^{-z} \sum_{i: J_{i} \neq \varnothing} \theta_{i}\left|\left\{l \in\left(1, \ldots, N_{i}\right): h_{i}^{(l)}=h\right\}\right|, \quad h=1, \ldots, q-1 .
$$

Это утверждение вытекает из соотношения (13).

Пусть выполняется случай (Б). Относительно подмножества $S \subseteq\{1, \ldots, n\}$ индексов переменных, не вошедших ни в одно уравнение системы (4), определим множество векторов

$$
\begin{gathered}
X_{g\left(x^{\circ}\right)}(S)=\left\{\left(x_{1}, \ldots, x_{n}\right): x_{s}=g\left(x_{s}^{\circ}\right) \forall s \notin S ;\right. \\
\left.x_{s} \in\{0, \ldots, q-1\} \forall s \in S\right\} .
\end{gathered}
$$

Теорема 2. Пусть имеет место случай (Б) и выполняется условие 1 теоремы 1. Пусть, кроме того, при $n \rightarrow \infty$

2) доля $\theta_{i}(n)=n_{i} / n$ координат в истинном решении $x^{\circ}$, принимающих значение $i$, заключена в границах $0<\delta \leqslant \theta_{i}(n) \leqslant 1-\delta<1$, $i=0, \ldots, q-1$;

3) величины $T(r)$ связаны с числам неизвестных $n$ соотношениями 3.1) $T(r) / n \rightarrow \infty, r=1, \ldots, R$,

3.2) $\sum_{r=1}^{R} T(r) d(r)=n(\ln n+z), z=O(1)$.

Tozda

1) $n p u n \rightarrow \infty$

$$
\mathbf{P}\left\{\xi=|G| q^{m}\right\}=\frac{e^{-z m}}{m !} \exp \left\{-e^{-z}+o(1)\right\}, \quad m=0,1,2, \ldots ;
$$

2) npu $n \rightarrow \infty$

$$
\mathbf{P}\left\{\Xi=\bigcup_{g \in G} X_{g\left(x^{\circ}\right)}(S)\right\} \rightarrow 1
$$

3) если $\left|G_{r}\right|=1, r=1, \ldots, R$, то утверждения 1, 2 выполнялотся независимо от условия 3.1 . 
Обозначим $\tilde{\lambda}_{r}(i, j)$ величину, полученную по формуле (8) после замены в ней постоянных параметров $\theta_{i}, i=0, \ldots, q-1$, на переменные $\theta_{i}(n)$.

Пусть $q=2$. Относительно произвольного подмножества $S \subseteq\{1, \ldots, n\}$ определим множество векторов

$$
X_{g\left(x^{\circ}\right)}(S)=\left\{x=\left(x_{1}, \ldots, x_{n}\right): x_{s}=g\left(x_{s}^{o}\right) \forall s \notin S ; x_{s} \in\{0,1\} \forall s \in S\right\} .
$$

Теорема 3. ПІусть $q=2$, выполняется условие 1 теоремы 1 (это условие принимает вид: $\varphi_{r} \not \equiv$ const,$\left.\forall \varphi_{r} \in \Phi\right)$ ) $и$ nрu $n \rightarrow \infty$

2) выполняется условие 2 теоремы 2;

3) величины $T(r)$ связаны с величиной $n$ соотношениями

3.1) $T(r) / n \rightarrow \infty, r=1, \ldots, R$,

3.2) $\sum_{r=1}^{R} T(r)\left(d(r)-\tilde{\lambda}_{r}(0,1)\right)=n(\ln n+z), z=O(1)$.

Toгda

1) $n p u n \rightarrow \infty$

$$
\mathbf{P}\left\{\xi=|G| 2^{m}\right\}=\frac{e^{-m z}}{m !} \exp \left\{-e^{-z}+o(1)\right\}, \quad m=0,1,2, \ldots ;
$$

2) $n p u n \rightarrow \infty$

$$
\mathbf{P}\left\{\exists S: \Xi=\bigcup_{g \in G} X_{g\left(x^{\circ}\right)}(S)\right\} \rightarrow 1
$$

если выполняется случай (Б) $u$, следовательно, $\tilde{\lambda}_{r}(0,1)=0$, $\boldsymbol{r}=1, \ldots, R$, то множество $S$ состоит из индексов переменных, не вошедиих ни в одно уравнение системы;

3) если $\left|G_{r}\right|=1, r=1, \ldots, R$, то утверждения 1,2 выполняются независимо от условия 3.1 .

3 а м е ч н и е 1 . Вариант теоремы 3 для случая $\left|G_{r}\right|=1, r=$ $1, \ldots, R$, доказан в работе [3].

3 а м е ч а и е 2. Условие 3.3 теоремы 1 с учетом условия 3.2 эквивалентно соотношению

$$
\sum_{r: \lambda_{r}^{*}=0} T(r) d(r)+T\left[\sum_{r: \lambda_{r}^{*}>0} t(r) d(r)-\lambda^{*}\right]=n(\ln n+z+o(1)) .
$$

Это соотношение является корректным (выполнимо, в частности, в случае $\left.\left\{r: \lambda_{r}^{*}=0\right\}=\varnothing\right)$, если

$$
\lambda^{*}<\sum_{r: \lambda_{r}^{*}>0} t(r) d(r)
$$

Покажем, что строгое неравенство (19) обеспечивается условиями 1,2 теоремы 1. Действительно, из формул (9) вытекает:

$$
\lambda^{*} \leqslant \sum_{r: \lambda_{r}^{*}>0} t(r) \max _{i, j} \lambda_{r}(i, j) .
$$


Для величины $\pi_{r}^{(k)}\left(i, j ; d_{0}, \ldots, d_{q-1}\right)$, согласно ее определению (см. (5) и ниже), выполняется неравенство

$$
\pi_{r}^{(k)}\left(i, j ; d_{0}, \ldots, d_{q-1}\right) \leqslant \frac{(d(r)-1) !}{d_{0} ! \cdots d_{q-1} !} .
$$

Поэтому с учетом (7), (8) имеем

$$
\max _{i, j} \lambda_{r}(i, j) \leqslant d(r)
$$

Если для функции $\varphi_{r}$ и любых элементов $i, j, i \neq j$, найдутся указанные в условии 1 теоремы 1 векторы $y^{\prime}, y^{\prime \prime}$, то при некотором наборе значений $d_{0}, \ldots, d_{q-1}, d_{0}+\cdots+d_{q-1}=d(r)-1$, в (21) будет выполняться строгое неравенство. Из этого неравенства и (7), (8) следует строгое неравенство в (22), из которого с учетом (20) следует (19).

\section{2. Вспомогательные утверждения}

Утверждение 1. Пусть функиия $\varphi_{r} \in \Phi$ обладает свойством из условия 1 теоремы 1, тогда для любого вырожденного (не взаимно однозначного) отображения $g^{\prime}:\{0, \ldots, q-1\} \longrightarrow\{0, \ldots, q-1\}$ найдется вектор у такой, что $\varphi_{r}(y) \neq \varphi_{r}\left(g^{\prime}(y)\right), g^{\prime}(y)=\left(g^{\prime}\left(y_{1}\right), \ldots, g^{\prime}\left(y_{d(r)}\right)\right)$.

Д ок аз ат е л с т в о. Предположим противное, а именно: сушествует вырожденное отображение $g^{\prime}$ такое, что $\varphi_{r}(y) \equiv \varphi_{r}\left(g^{\prime}(y)\right)$. В силу вырожденности $g^{\prime}$ для некоторых элементов $i, j \in\{0, \ldots, q-1\}$, $i \neq j$, выполняется равенство $g^{\prime}(i)=g^{\prime}(j)$. Тогда для каждой пары векторов $y^{\prime}, y^{\prime \prime}$, вида (6), где $y_{k}^{\prime}=i, y_{k}^{\prime \prime}=j, k=1, \ldots, d(r)$, должно выполняться равенство $\varphi_{r}\left(y^{\prime}\right)=\varphi_{r}\left(y^{\prime \prime}\right)$, так как $\varphi_{r}\left(y^{\prime}\right)=\varphi_{r}\left(g^{\prime}\left(y^{\prime}\right)\right)$, $\varphi_{r}\left(y^{\prime \prime}\right)=\varphi_{r}\left(g^{\prime}\left(y^{\prime \prime}\right)\right)$, и $g^{\prime}\left(y^{\prime}\right)=g^{\prime}\left(y^{\prime \prime}\right)$, согласно равенству $g^{\prime}(i)=g^{\prime}(j)$. Утверждение 1 доказано.

Пусть $\mathbf{E} \boldsymbol{\xi}$ - математическое ожидание числа $\boldsymbol{\xi}$.

Лемма 1. Пусть выполняются условия теоремы 1. Тогда при $n \rightarrow \infty$

$$
\mathbf{E} \xi=|G| \exp \left\{\sum_{i=0}^{q-1} \theta_{i} h_{i} e^{-z}+o(1)\right\}
$$

где $h_{i}$ - иисло әлементов в множестве $J_{i}$.

Лемма 2. Пусть выполняются условия теоремы 2. Тогда

1) $\quad$ npu $n \rightarrow \infty \quad \mathbf{E} \xi=|G| \exp \left\{(q-1) e^{-z}+o(1)\right\}$

2) если $\left|G_{r}\right|=1, r=1, \ldots, R$, то утверждение 1 выполняется независимо от условия 3.1 теоремы. 
Лемма 3. Пусть $q=2$ и выполняются условия теоремы 3. Тогда

1) $n p u n \rightarrow \infty \mathbf{E} \xi=|G| \exp \left\{e^{-z}+o(1)\right\}$;

2) если $\left|G_{r}\right|=1, r=1, \ldots, R$, то утверждение 1 выполняется независимо от условия 3.1 теоремьт.

2.1. Д ок аз а т ельс т во ле м м ы. Обозначим $\rho\left(x^{\prime}, x^{\prime \prime}\right)$ расстояние Хемминга между векторами $x^{\prime}=\left(x_{1}^{\prime}, \ldots, x_{n}^{\prime}\right)$ и $x^{\prime \prime}=$ $\left(x_{1}^{\prime \prime}, \ldots, x_{n}^{\prime \prime}\right)$ :

$$
\rho\left(x^{\prime}, x^{\prime \prime}\right)=\sum_{k=1}^{n} I\left(x_{k}^{\prime} \neq x_{k}^{\prime \prime}\right), \quad I\left(x_{k}^{\prime} \neq x_{k}^{\prime \prime}\right)= \begin{cases}1, \text { если } & x_{k}^{\prime} \neq x_{k}^{\prime \prime} ; \\ 0, \text { если } & x_{k}^{\prime}=x_{k}^{\prime \prime}\end{cases}
$$

Определим $\varepsilon$-окрестность вектора $x^{\prime}$ как множество векторов

$$
M_{\varepsilon}\left(x^{\prime}\right)=\left\{x: \rho\left(x^{\prime}, x^{\prime \prime}\right) \leqslant \varepsilon n\right\} .
$$

Без ограничения общности мы можем положить в условии 2 теоремы 1 , что $0<\delta \leqslant \theta_{i}(n) \leqslant 1-\delta<1$. Тогда при любых подстановках $g_{1}, g_{2} \in G, g_{1} \neq g_{2}$,

$$
\rho\left(g_{1}\left(x^{\circ}\right), g_{2}\left(x^{\circ}\right)\right)=\rho\left(x^{\circ}, g_{1}^{-1} g_{2}\left(x^{\circ}\right)\right) \geqslant n \min _{i \in\{0, \ldots, q-1\}} \theta_{i} \geqslant n \delta .
$$

Выберем $\varepsilon<\delta / 2$. При этом условии $M_{\varepsilon}\left(g_{1}\left(x^{\circ}\right)\right) \cap M_{\varepsilon}\left(g_{2}\left(x^{\circ}\right)\right)=\varnothing$, $\forall g_{1}, g_{2} \in G, g_{1} \neq g_{2}$, и математическое ожидание $\mathbf{E} \xi$ можно представить в следуюшем виде:

$$
\begin{aligned}
& \mathbf{E} \xi=\Sigma_{1}+\Sigma_{2}, \\
& \Sigma_{1}=\sum_{g \in G} \sum_{x \in M_{\varepsilon}\left(g\left(x^{\circ}\right)\right)} \prod_{r=1}^{R}\left[P_{r}(x)\right]^{T(r)}, \\
& \Sigma_{2}\left.=\sum_{x \notin \cup_{g} \in G} M_{\varepsilon}\left(g\left(x^{\circ}\right)\right)\right) \\
& \prod_{r=1}^{R}\left[P_{r}(x)\right]^{T(r)},
\end{aligned}
$$

где $P_{r}(x)$ - вероятность того, что вектор $x$ удовлетворяет одному уравнению системы (4), связанному с функцией $\varphi_{r}$.

Оценим суммы $\Sigma_{1}, \Sigma_{2}$ из разложения (25). Согласно равенству $\rho\left(x^{\circ}, x\right)=\rho\left(g\left(x^{\circ}\right), g(x)\right)$, имеем $M_{\varepsilon}\left(g\left(x^{\circ}\right)\right)=\left\{g(x): x \in M_{\varepsilon}\left(x^{\circ}\right)\right\}$. Кроме того, $P_{r}(g(x))=P_{r}(x) \forall g \in G$, так как по определению группы $G$ векторы $x$ и $g(x)$ одновременно удовлетворяют или не удовлетворяют одному уравнению системы с функцией $\varphi_{r}$. Поэтому для внутренней суммы в слагаемом $\Sigma_{1}$ из разложения (25) получаем

$$
\begin{aligned}
\sum_{x \in M_{\varepsilon}\left(g\left(x^{\circ}\right)\right)} \prod_{r=1}^{R}\left[P_{r}(x)\right]^{T(r)} & =\sum_{x \in M_{\varepsilon}\left(x^{\circ}\right)} \prod_{r=1}^{R}\left[P_{r}(g(x))\right]^{T(r)} \\
& =\sum_{x \in M_{\varepsilon}\left(x^{\circ}\right)} \prod_{r=1}^{R}\left[P_{r}(x)\right]^{T(r)}
\end{aligned}
$$


Следовательно,

$$
\Sigma_{1}=|G| \Sigma_{1}^{*}, \quad \Sigma_{1}^{*}=\sum_{x \in M_{\varepsilon}\left(x^{\circ}\right)} \prod_{r=1}^{R}\left[P_{r}(x)\right]^{T(r)}
$$

Положим

$$
n_{i j}\left(x^{\circ}, x\right)=\left|\left\{s: x_{s}^{\circ}=i, x_{s}=j\right\}\right| .
$$

По определению расстояния Хемминга имеем

$$
\rho\left(x^{\circ}, x\right)=\sum_{i, j: i \neq j} n_{i j}\left(x^{\circ}, x\right)
$$

Ясно, что все векторы $\boldsymbol{x}$, имеющие одинаковый набор чисел

$$
n_{i j}\left(x^{\circ}, x\right)=n_{i j}, \quad i, j=\overline{0, q-1},
$$

имеют одинаковую вероятность

$$
P_{r}(x)=P_{r}\left[n_{i j} ; i, j=\overline{0, q-1}\right] .
$$

Количество векторов $x$, которым соответствует фиксированный набор чисел (27), равно $\prod_{i=0}^{q-1} n_{i} !\left[n_{i, 0} ! \cdots n_{i, q-1} !\right]^{-1}$. Следовательно, сумму (26) можно переписать в следующем виде

$$
\Sigma_{1}^{*}=\sum_{n_{i j}: \sum_{i \neq j} n_{i j \leqslant \varepsilon n}} D\left[n_{i j} ; i, j=\overline{0, q-1}\right],
$$

где

$$
\begin{aligned}
\mathcal{D} & {\left[n_{i j} ; i, j=\overline{0, q-1}\right] } \\
& =\prod_{i=0}^{q-1} \frac{n_{i} !}{n_{i, 0} ! \cdots n_{i, q-1} !} \prod_{r=1}^{R}\left(P_{r}\left[n_{i j} ; i, j=\overline{0, q-1}\right]\right)^{T(r)}
\end{aligned}
$$

и суммирование проводится по всем значениям величин $n_{i j}$, удовлетворяющим условию $\sum_{i \neq j} n_{i j}=\sum_{i, j: i \neq j} n_{i j} \leqslant \varepsilon n$.

Обозначим $v=v(x)$ число координат вектора $x$ из множества $\left\{x_{s} \in\right.$ $\left.\left(x_{1}, \ldots, x_{n}\right): x_{s} \neq x_{s}^{\circ}\right\}$, входящих в уравнение

$$
\varphi_{r}\left(x_{s_{1}(t)}, \ldots, x_{s_{d(r)}}(t)\right)=\varphi_{r}\left(x_{s_{1}(t)}^{\circ}, \ldots, x_{s_{d(r)}}^{\circ}(t)\right) .
$$

Для вероятности (28), согласно формуле полной вероятности, получим

$$
\begin{aligned}
P_{r}(x)= & \mathbf{P}\{v=0\} P_{r}(x \mid v=0)+\mathbf{P}\{v=1\} P_{r}(x \mid v=1) \\
& +\sum_{v^{\prime} \geqslant 2} \mathbf{P}\left\{v=v^{\prime}\right\} P_{r}\left(x \mid v=v^{\prime}\right)
\end{aligned}
$$


где через $P_{r}\left(x \mid v=v^{\prime}\right)$ обозначена вероятность того, что при условии $v(x)=v^{\prime}$ вектор $x$ удовлетворяет уравнению (30).

Пусть $\sum_{i \neq j} n_{i j}=o(n)$. Последовательно раскрывая слагаемые в правой части равенства (31), найдем

$$
\begin{aligned}
P_{r}(x)= & P_{r}\left[n_{i j} ; i, j=\overline{0, q-1}\right]=\left[\left(\begin{array}{c}
n \\
d(r)
\end{array}\right) \frac{d(r) !}{\left|\Sigma_{\varphi_{r}}\right|}\right]^{-1} \\
\times & {\left[\left(\begin{array}{c}
\sum_{i=0}^{q-1} n_{i i} \\
d(r)
\end{array}\right) \frac{d(r) !}{\left|\Sigma_{\varphi_{r}}\right|}\right.} \\
& +\sum_{i \neq j} n_{i j} \sum_{\substack{d_{0}, \ldots, d_{q-1} \\
d_{0}+\cdots+d_{q-1}=d(r)-1}} \prod_{i=0}^{q-1}\left(\begin{array}{c}
n_{i i} \\
d_{i}
\end{array}\right) \pi_{r}\left(i, j ; d_{0}, \ldots, d_{q-1}\right) \frac{\prod_{i=0}^{q-1} d_{i} !}{\left|\Sigma_{\varphi_{r}}\right|} \\
& \left.+O\left(n^{d-2}\left(\sum_{i \neq j} n_{i j}\right)^{2}\right)\right]
\end{aligned}
$$

где величина $\pi_{r}\left(i, j ; d_{0}, \ldots, d_{q-1}\right)$ определяется формулой (5). Выражение (32) перепишем в следующем виде:

$$
\begin{aligned}
& P_{r}\left[n_{i j} ; i, j=\overline{0, q-1}\right] \\
& \quad=1-\frac{1}{n} \sum_{i \neq j} n_{i j}\left(d(r)-\tilde{\lambda}_{r}(i, j)\right)+O\left(\left(\sum_{i \neq j} \frac{n_{i j}}{n}\right)^{2}\right)
\end{aligned}
$$

где величина $\tilde{\lambda}_{r}(i, j)$ получена по формуле (8) после замены в ней фиксированных параметров $\theta_{i}, i=0, \ldots, q-1$, на переменные $\theta_{i}(n)$.

Разобьем сумму (29) на две части

$$
\Sigma_{1}^{*}=\Sigma_{1,1}^{*}+\Sigma_{1,2}^{*}
$$

полагая

$$
\begin{aligned}
& \Sigma_{1,1}^{*}=\sum_{n_{i j}: 0 \leqslant \sum_{i \neq j} n_{i j \leqslant n^{1 / 3}}} D\left[n_{i j} ; i, j=\overline{0, q-1}\right], \\
& \sum_{n_{i j}: n^{1 / 3}<\sum_{i \neq j} n_{i j} \leqslant \varepsilon n} D\left[n_{i j} ; i, j=\overline{0, q-1}\right],
\end{aligned}
$$

и оценим каждую часть по отдельности.

В области $0 \leqslant \sum_{i \neq j} n_{i j} \leqslant n^{1 / 3}$ имеем оценки

$$
\prod_{i=0}^{q-1} \frac{n_{i} !}{n_{i, 0} ! \cdots n_{i, q-1} !}=\exp \left\{\sum_{i \neq j} n_{i j} \ln n+o(1)\right\} \prod_{i=0}^{q-1} \prod_{j: j \neq i} \frac{\left(\theta_{i}(n)\right)^{n_{i j}}}{n_{i j} !}
$$




$$
\begin{aligned}
& \prod_{r=1}^{R}\left(P_{r}\left[n_{i j} ; i, j=\overline{0, q-1}\right]\right)^{T(r)} \\
& \quad=\exp \left\{-\sum_{i \neq j} n_{i j} \frac{1}{n} \sum_{r=1}^{R} T(r)\left(d(r)-\tilde{\lambda}_{r}(i, j)\right)+o(1)\right\} .
\end{aligned}
$$

Положим

$$
\sigma(i, j)=\frac{1}{n} \sum_{r=1}^{R} T(r)\left(d(r)-\tilde{\lambda}_{r}(i, j)\right)-\ln n
$$

Согласно условиям 2, 3.2, 3.3 теоремы 1 ,

$$
\begin{aligned}
\sigma(i, j) & =\frac{1}{n}\left(\sum_{r=1}^{R} T(r) d(r)-T\left(\lambda^{*}-\Delta(i, j)\right)+o(n)\right)-\ln n \\
& =\frac{T}{n} \Delta(i, j)+z+o(1)
\end{aligned}
$$

где $\Delta(i, j)=0,(i, j) \in M$ и $\Delta(i, j)>0,(i, j) \notin M$, множество $M$ определено формулами (9). Следовательно, при условии 3.1 теоремы 1

$$
\sigma(i, j) \rightarrow \infty, \quad(i, j) \notin M
$$

Поэтому с учетом неравенств $\Psi\left(n^{1 / 3} / q^{2}\right) \leqslant \Sigma_{1,1}^{*} \leqslant \Psi\left(n^{1 / 3}\right)$, где

$$
\Psi(z)=\prod_{i=0}^{q-1} \prod_{j: j \neq i} \sum_{n_{i j}=0}^{z} \exp \left\{-n_{i j} \sigma(i, j)+o(1)\right\} \frac{\left(\theta_{i}(n)\right)^{n_{i j}}}{n_{i j} !}
$$

а также с учетом соотношений (36), (37) и условия 2 теоремы 1 найдем

$$
\begin{aligned}
\Sigma_{1,1}^{*} & \approx \prod_{i: J_{i} \neq \varnothing} \prod_{j: j \in J_{i}} \sum_{n_{i j}=0}^{\infty} \exp \left\{-n_{i j} z\right\} \frac{\theta_{i}^{n_{i j}}}{n_{i j} !} \\
& =\prod_{i: J_{i} \neq \varnothing} \exp \left\{\theta_{i} h_{i} e^{-z}\right\}=\prod_{i=0}^{q-1} \exp \left\{\theta_{i} h_{i} e^{-z}\right\}
\end{aligned}
$$

где $h_{i}$ - число элементов в множестве $J_{i}$.

Опеним теперь сумму $\Sigma_{1,2}^{*}$ из разложения (34). Из представления (33) вытекает

$$
\prod_{r=1}^{R}\left(P_{r}\left[n_{i j} ; i, j=\overline{0, q-1}\right]\right)^{T(r)} \leqslant \exp \left\{-\frac{\sigma}{n} \delta(\varepsilon) \sum_{i \neq j} n_{i j}\right\}
$$


где $\sigma=\sum_{r=1}^{R} T(r) d(r)-T \lambda^{*}, \lim _{\varepsilon \rightarrow 0} \delta(\varepsilon)=1$. Пользуясь этой оценкой и неравенствами

$$
\begin{aligned}
\prod_{i=0}^{q-1} \frac{n_{i} !}{n_{i, 0} ! \cdots n_{i, q-1} !} & \leqslant \prod_{i=0}^{q-1} \frac{n_{i} !}{n_{i i} !\left(n_{i}-n_{i i}\right) !}(q-1)^{n_{i}-n_{i i}} \\
& \leqslant(q-1)^{\sum_{i \neq j} n_{i j}}\left(\begin{array}{c}
n \\
\sum_{i \neq j} n_{i j}
\end{array}\right)
\end{aligned}
$$

а также полагая $H(u)=-u \ln u-(1-u) \ln (1-u)$, получаем

$$
\begin{aligned}
\Sigma_{1,2}^{*} \leqslant \sum_{n_{i j}: n^{1 / 3}<\sum_{i \neq j} n_{i j} \leqslant \varepsilon n} \exp \{- & {[\sigma \delta(\varepsilon)-n \ln (q-1)] u } \\
& +n H(u)+O(\ln n)\}\left.\right|_{u=\sum_{i \neq j} n_{i j} / n} .
\end{aligned}
$$

Функция $H(u)$ в области $0<u<\varepsilon$ выпукла вверх, поэтому с учетом условия 3.3 теоремы 1 имеем

$$
\begin{aligned}
\Sigma_{1,2}^{*} & \leqslant\left. n^{q(q-1)} \exp \{-\sigma(\delta(\varepsilon)+o(1)) u+n H(u)+O(\ln n)\}\right|_{u=n^{1 / 3} / n} \\
& \leqslant n^{q(q-1)} \exp \left\{-\left(\delta(\varepsilon)-\frac{2}{3}+o(1)\right) n^{1 / 3} \ln n+O\left(n^{1 / 3}\right)\right\}=o(1),
\end{aligned}
$$

если значение $\varepsilon$ достаточно мало.

Нам осталось оценить сумму $\Sigma_{2}$ из разложения (25). Покажем, что для любого вектора $x \notin \cup_{g \in G} M_{\varepsilon}\left(g\left(x^{\circ}\right)\right)$ найдется функция $\varphi_{r} \in \Phi$ такая, что

$$
P_{r}(x)=P_{r}\left[n_{i j} ; i, j=\overline{0, q-1}\right] \leqslant 1-\alpha(\varepsilon),
$$

где $0<\alpha(\varepsilon)=$ const. Из неравенства (40) будет следовать оценка

$$
\Sigma_{2} \leqslant q^{n}(1-\alpha(\varepsilon))^{T^{\prime}}=o(1)
$$

где $T^{\prime}=\min _{r} T(r)$, и $T^{\prime} / n \rightarrow \infty$, согласно условию 3.1 теоремы 1 .

Собирая вместе (25), (26), (34) и оценки (38), (39), (41), получим (23).

Пусть набор чисел $n_{i j}\left(x^{\circ}, x\right)=n_{i j}, i, j=0, \ldots, q-1$, удовлетворяет условию

$$
\sum_{i \neq j} n_{i j}=\sum_{i=0}^{q-1} \sum_{j: j \neq i} n_{i j} \geqslant \varepsilon n .
$$

Из этого условия слепует, что существует (единственный) набор индексов $i_{1}, \ldots, i_{k}, 1 \leqslant k \leqslant q$, которому соответствуют неравенства

$$
\begin{gathered}
\sum_{j: j \neq i_{1}} n_{i_{1}, j}>\frac{\varepsilon n}{q}, \ldots, \sum_{j: j \neq i_{k}} n_{i_{k}, j}>\frac{\varepsilon n}{q} ; \\
\sum_{j: j \neq i} n_{i j} \leqslant \frac{\varepsilon n}{q}, \quad i \notin\left\{i_{1}, \ldots, i_{k}\right\} .
\end{gathered}
$$


В свою очередь, из неравенств (42) вытекает, что найдется набор индексов $j_{1}, \ldots, j_{k}, j_{1} \neq i_{1}, \ldots, j_{k} \neq i_{k}$, для которого

$$
n_{i_{1}, j_{1}}>\frac{\varepsilon n}{q(q-1)}, \ldots, n_{i_{k}, j_{k}}>\frac{\varepsilon n}{q(q-1)},
$$

а из неравенств (43) с учетом соотношений $\sum_{j=0}^{q-1} n_{i j}=n_{i}=\theta_{i}(n) n$ следует:

$$
n_{i i}=n_{i}-\sum_{j: j \neq i} n_{i j} \geqslant\left(\theta_{i}(n)-\frac{\varepsilon}{q}\right) n, \quad i \notin\left\{i_{1}, \ldots, i_{k}\right\} .
$$

Сопоставим системе неравенств (44), (45) отображение $g^{\prime}:\{0, \ldots$, $q-1\} \longrightarrow\{0, \ldots, q-1\}$, определяемое условиями

$$
g^{\prime}\left(i_{1}\right)=j_{1}, \ldots, g^{\prime}\left(i_{k}\right)=j_{k} ; \quad g^{\prime}(i)=i, \quad i \notin\left\{i_{1}, \ldots, i_{k}\right\}
$$

и рассмотрим два возможных случая: (В) $g^{\prime} \notin G,(\Gamma) g^{\prime} \in G$.

(В) Пусть $g^{\prime} \notin G$. Тогда для некоторой функции $\varphi_{r} \in \Phi$ найдется вектор $y$ такой, что $\varphi_{r}(y) \neq \varphi_{r}\left(g^{\prime}(y)\right)$. Действительно, если отображение $g^{\prime}$ является вырожденным, то указанный вектор $y$ найдется согласно утверждению 1 , в противном случае он найдется согласно определению группы $G$.

Пусть вектор $y$ содержит $d_{w}$ координат, принимающих значение $w=0, \ldots, q-1$. Тогда вероятность того, что упорядоченная выборка координат $\left(x_{s_{1}(t)}, \ldots, x_{s_{d(r)}(t)}\right)$ из вектора $x=\left(x_{1}, \ldots, x_{n}\right)$, соответствующая уравнению

$$
\varphi_{r}\left(x_{s_{1}(t)}, \ldots, x_{s_{d(r)}(t)}\right)=\varphi_{r}\left(x_{s_{1}(t)}^{\mathrm{o}}, \ldots, x_{s_{d(r)}(t)}^{\mathrm{o}}\right)
$$

образует вектор $y$, равна

$$
P=\prod_{\nu=1}^{k}\left(\begin{array}{c}
n_{i_{\nu}, j_{\nu}} \\
d_{i_{\nu}}
\end{array}\right) d_{i_{\nu}} ! \prod_{i \notin\left\{i_{1}, \ldots, i_{k}\right\}}\left(\begin{array}{c}
n_{i i} \\
d_{i}
\end{array}\right) \frac{d_{i} !}{\left|\Sigma_{\varphi_{r}}\right|}\left[\left(\begin{array}{c}
n \\
d(r)
\end{array}\right) \frac{d(r) !}{\left|\Sigma_{\varphi_{r}}\right|}\right]^{-1}
$$

Отсюда с учетом (44), (45) вытекает

$$
\begin{aligned}
& 1-P_{r}\left[n_{i j} ; i, j=\overline{0, q-1}\right] \\
& \quad \geqslant P=(1+o(1))\left(\frac{\varepsilon}{q(q-1)}\right)^{k} \prod_{i \notin\left\{i_{1}, \ldots, i_{k}\right\}}\left(\theta_{i}-\frac{\varepsilon}{q}\right) .
\end{aligned}
$$

Следовательно, существует функция $\varphi_{r} \in \Phi$, для которой выполняется оценка (40) (при достаточно больших значениях $n$ ).

(Г) Рассмотрим теперь второй случай:

$$
g^{\prime}=g \in G \text {. }
$$


Выберем $\varepsilon$ такое, что

$$
\theta_{i_{\nu}}(n)-\frac{\varepsilon}{q(q-1)}>\frac{\varepsilon}{q(q-1)}, \quad \nu=\overline{1, k}
$$

и предположим, что выполняются более жесткие по сравнению с неравенствами (44) ограничения

$$
n_{i_{1}, j_{1}} \geqslant\left(\theta_{i_{1}}(n)-\frac{\varepsilon}{q(q-1)}\right) n, \ldots, n_{i_{k}, j_{k}} \geqslant\left(\theta_{i_{k}}(n)-\frac{\varepsilon}{q(q-1)}\right) n .
$$

Положим $n_{i j}^{(g)}=n_{i j}^{(g)}\left(x^{\circ}, x\right)=\left|\left\{s: g\left(x_{s}^{o}\right)=i, x_{s}=j\right\}\right|$ и с учетом (49) и (45) получим, что

$$
\begin{aligned}
n_{i_{\nu}, j_{\nu}} & =n_{e\left(i_{\nu}\right), j_{\nu}}^{(e)}=n_{g\left(i_{\nu}\right), j_{\nu}}^{(g)}=n_{j_{\nu}, j_{\nu}}^{(g)} \geqslant\left(\theta_{i_{\nu}}(n)-\frac{\varepsilon}{q(q-1)}\right) n, \quad \nu=\overline{1, k} \\
n_{i i} & =n_{e(i), i}^{(e)}=n_{g(i), i}^{(g)}=n_{i i}^{(g)} \geqslant\left(\theta_{i}(n)-\frac{\varepsilon}{q}\right) n, \quad i \notin\left\{i_{1}, \ldots, i_{k}\right\}
\end{aligned}
$$

где $e-$ единичный элемент группы $G$. Следовательно,

$$
\begin{aligned}
\rho\left(g\left(x^{\circ}\right), x\right) & =n-\sum_{j_{\nu} \in\left\{i_{1}, \ldots, i_{k}\right\}} n_{j_{\nu}, j_{\nu}}^{(g)}-\sum_{i \notin\left\{i_{1}, \ldots, i_{k}\right\}} n_{i i}^{(g)} \\
& \leqslant \varepsilon n\left(1-\frac{k}{q}+\frac{k}{q(q-1)}\right) \leqslant \varepsilon n, \quad \forall q \geqslant 2 .
\end{aligned}
$$

Значит, при условии (49) вектор $x$ принадлежит $\varepsilon$-окрестности решения $g\left(x^{\circ}\right)$ и не должен учитываться в сумме $\Sigma_{2}$ из разложения (25). Поэтому, по крайней мере, одно из неравенств (49) должно нарушаться.

Пусть

$$
n_{i_{\nu}, j_{\nu}}<\left(\theta_{i_{\nu}}(n)-\frac{\varepsilon}{q(q-1)}\right) n, \quad \nu \in\{1, \ldots, k\}
$$

С учетом (50) и равенств $\sum_{j=0}^{q-1} n_{i_{\nu}, j}=\sum_{j: j \neq j_{\nu}} n_{i_{\nu}, j}+n_{i_{\nu}, j_{\nu}}=\theta_{i_{\nu}}(n) n$ получим, что найдется элемент $j \in\{0, \ldots, q-1\} \backslash j_{\nu}$, для которого

$$
n_{i_{\nu}, j}>\left[\theta_{i_{\nu}}(n) n-\left(\theta_{i_{\nu}}(n)-\frac{\varepsilon}{q(q-1)}\right) n\right] \frac{1}{q-1}=\frac{\varepsilon}{q(q-1)^{2}} n .
$$

Согласно (44) и (51), имеем

$$
\begin{aligned}
n_{i_{1}, j_{1}}, \ldots, n_{i_{\nu-1}, j_{\nu-1}} & \geqslant \frac{\varepsilon}{q(q-1)} n \\
n_{i_{\nu}, j} & \geqslant \frac{\frac{\varepsilon}{q(q-1)^{2}} n}{\varepsilon} \\
n_{i_{\nu+1}, j_{\nu+1}}, \ldots, n_{i_{k}, j_{k}} & \geqslant \frac{q(q-1)}{q(q}
\end{aligned}
$$


где $\nu \in\{1, \ldots, k\} ; j \in\{0, \ldots, q-1\} \backslash j_{\nu}$. При этом остаются в силе ограничения (45).

Сопоставим ограничениям (52) и (45) отображение $g^{\prime \prime}:\{0, \ldots$, $q-1\} \longrightarrow\{0, \ldots, q-1\}$, определяемое равенствами

$$
\begin{aligned}
g^{\prime \prime}\left(i_{1}\right) & =j_{1}, \ldots, g^{\prime \prime}\left(i_{\nu-1}\right)=j_{\nu-1} \\
g^{\prime \prime}\left(i_{\nu}\right) & =j \in\{0, \ldots, q-1\} \backslash j_{\nu} \\
g^{\prime \prime}\left(i_{\nu+1}\right) & =j_{\nu+1}, \ldots, g^{\prime \prime}\left(i_{k}\right)=j_{k} \\
g^{\prime \prime}(i) & =i, \quad i \notin\left\{i_{1}, \ldots, i_{k}\right\}
\end{aligned}
$$

Отображение $g^{\prime \prime}$ отличается от заданного равенствами (46) взаимно однозначного по условию (48) отображения $g^{\prime}$ только одним переходом: $g^{\prime \prime}\left(i_{\nu}\right)=j \in\{0, \ldots, q-1\} \backslash j_{\nu}$. Следовательно, отображение $g^{\prime \prime}$ является вырожденным. Поэтому по условию 1 теоремы 1 и согласно утверждению 1 , для любой функции $\varphi_{r} \in \Phi$ найдется вектор $y$ такой, что $\varphi_{r}(y) \neq \varphi_{r}\left(g^{\prime \prime}(y)\right)$.

Теперь мы можем повторить рассуждения, использованные для вывода оценки (47) в случае (В) (см. (46) и ниже), и с учетом (45), (52) заменить в (47) один множитель, равный $\varepsilon / q(q-1)$, на величину $\varepsilon / q(q-1)^{2}$. Таким образом получим оценку (40) в случае $(\Gamma)$. Лемма 1 доказана.

2.2. Д о а за т е л с т в о л м м ы 2. Доказательство леммы 2 до формулы (35) повторяет доказательство леммы 1. Для величины (35) согласно условию 3.2 теоремы 2 имеем $\sigma(i, j)=z$. Используя это равенство, аналогично выводу оценки (38) получим

$$
\begin{aligned}
\Sigma_{1,1}^{*} & \approx \prod_{i=0}^{q-1} \prod_{j: j \neq i} \sum_{n_{i j}=0}^{\infty} \exp \left\{-n_{i j} z\right\} \frac{\theta_{i}^{n_{i j}}}{n_{i j} !} \\
& =\prod_{i=0}^{q-1} \exp \left\{(q-1) \theta_{i} e^{-z}\right\}=\exp \left\{(q-1) e^{-z}\right\}
\end{aligned}
$$

и эта оценка не зависит от условия 3.1 теоремы 2 (условие 3.1 использовалось в доказательстве леммы 1 при обосновании оценки (38) и при обосновании оценки (41)).

Совершенно аналогично показывается (с учетом условия 3.2 теоремы 2 вместо условия 3.3 теоремы 1), что выполняется оценка (39) и эта оценка не зависит от условия 3.1 .

Без каких-либо изменений (с учетом условия 3.1 ) доказывается оценка (41) и, таким образом, завершается доказательство утверждения 1 леммы 2.

Для доказательства утверждения 2 леммы 2 осталось показать, что в случае $\left|G_{\varphi}\right|=1, r=1, \ldots, R$, независимо от условия 3.1 выполняется оценка вида (41). Пусть $\left|G_{\varphi}\right|=1, r=1, \ldots, R$, тогда неравенство (40) 
выполняется для любого вектора $x \notin \cup_{g \in G} M_{\varepsilon}\left(g\left(x^{\circ}\right)\right)$ и для любой функции $\varphi_{r} \in \Phi$, так как для любой функции $\varphi_{r} \in \Phi$ найдется вектор $y$ такой, что $\varphi_{r}(y) \neq \varphi_{r}\left(g^{\prime}(y)\right)$, где отображение $g^{\prime} \notin G$ определено равенствами (46). Поэтому

$$
\Sigma_{2} \leqslant q^{n}(1-\alpha(\varepsilon))^{T^{\prime \prime}}=o(1)
$$

где $T^{\prime \prime}=\max _{r} T(r)$ и $T^{\prime \prime} / n \rightarrow \infty$, согласно условию 3.2 теоремы 2 . Лемма 2 доказана.

2.3. Д оказат ел ьс т в о л м м ы 3. Доказательство леммы 3 фактически вытекает из доказательств лемм 1, 2. Следует только учесть, что при $q=2$ как в случае (А), так и в случае (Б) для величины $(35)$ имеем $\sigma(0,1)=z$. Поэтому независимо от условия 3.1 теоремы 3 выполняется оценка (54), где $q=2$.

\section{3. Доказательства теорем}

3.1. Доказательст в о те орем ы 1 . Обозначим $\eta$ число пар переменных $x_{s_{1}\left(i_{1}\right)}, x_{s_{2}\left(i_{2}\right)}$, где

$$
i_{1} \leqslant i_{2}, s_{1}(i)<s_{2}(i), \quad s_{1}\left(i_{1}\right), s_{2}\left(i_{2}\right) \in \bigcup_{i: J_{i} \neq \varnothing} \bigcup_{l=1}^{N_{i}} S_{i}^{(l)}
$$

содержащихся одновременно, по крайней мере, в одном уравнении системы. Пусть событие $B_{0}$ состоит в том, что $\eta=0$.

Для набора фиксированных неотрицательных целых чисел $\boldsymbol{m}_{i}^{(l)}$, где $i, l$ пробегают значения $i: J_{i} \neq \varnothing, l=1, \ldots, N_{i}$, определим событие $B_{0}\left(\left\{m_{i}^{(l)}\right\}\right)$, состоящее в том, что $\eta=0$ и, кроме того, выполняется система равенств $\left|S_{i}^{(l)}\right|=m_{i}^{(l)}$. Согласно определениям, имеем

$$
\begin{array}{r}
\mathbf{P}\left\{\Xi \supseteq \bigcup_{g \in G} X_{g\left(x^{\circ}\right)}\left(\left\{S_{i}^{(l)}\right\}\right) \mid B_{0}\right\}=1, \\
\mathbf{P}\left\{\xi \geqslant|G| \prod_{i: J_{i} \neq \varnothing} \prod_{l=1}^{N_{i}}\left(1+h_{i}^{(l)}\right)^{m_{i}^{(l)}} \mid B_{0}\left(\left\{m_{i}^{(l)}\right\}\right)\right\}=1,
\end{array}
$$

где $h_{i}^{(l)}=\left|J_{i}^{(l)}\right|$. Покажем теперь, что

$$
\mathbf{P}\left(B_{0}\left(\left\{m_{i}^{(l)}\right\}\right)\right)=\prod_{i: J_{i} \neq \varnothing} \prod_{l=1}^{N_{i}} \frac{\left(\theta_{i} e^{-z}\right)^{m_{i}^{(l)}}}{m_{i}^{(l)} !} \exp \left\{-\theta_{i} e^{-z}+o(1)\right\}
$$

Тогда с учетом (23)

$|G| \exp \left\{\sum_{i=0}^{q-1} \theta_{i} h_{i} e^{-z}+o(1)\right\}=\mathbf{E} \xi \geqslant \sum_{m_{i}^{(l)}} \mathbf{P}\left(B_{0}\left(\left\{m_{i}^{(l)}\right\}\right)\right) \mathbf{E}\left(\xi \mid B_{0}\left(\left\{m_{i}^{(l)}\right\}\right)\right)$ 
и, согласно (55), (56), правая часть этого неравенства не превосходит величины

$$
E^{\prime}=|G| \exp \left\{\sum_{i=0}^{q-1} \theta_{i} h_{i} e^{-z}+o(1)\right\}
$$

Отсюда вытекает, что

$$
\mathbf{P}\left\{\xi=|G| \prod_{i: J_{i} \neq \varnothing} \prod_{l=1}^{N_{i}}\left(1+h_{i}^{(l)}\right)^{m_{i}^{(l)}} \mid B_{0}\left(\left\{m_{i}^{(l)}\right\}\right)\right\}=1+o(1)
$$

Из (56), (57) следуют соотношения (13), (14).

Итак, нам осталось доказать соотношение (56). Оценим сначала вероятность $\mathbf{P}\left\{\left|S_{i}^{(l)}\right|=m_{i}^{(l)}\right\}, m_{i}^{(l)} \in\{0,1,2, \ldots\}$, выполнения системы равенств $\left|S_{i}^{(l)}\right|=m_{i}^{(l)}$, где $i, l$ пробегают значения $i: J_{i} \neq \varnothing, l=1, \ldots, N_{i}$.

Используя формулу включения и исключения, получим выражение вида

$$
\begin{aligned}
\mathbf{P} & \left\{\left|S_{i}^{(l)}\right|=m_{i}^{(l)}\right\} \\
& =\sum_{\mu_{i}^{(l)}} \prod_{i: J_{i} \neq \varnothing} \prod_{l=1}^{N_{i}}(-1)^{\mu_{i}^{(l)}}\left(\begin{array}{c}
m_{i}^{(l)}+\mu_{i}^{(l)} \\
\mu_{i}^{(l)}
\end{array}\right)\left(\begin{array}{c}
n_{i}-\sum_{p=1}^{l-1}\left(m_{i}^{(p)}+\mu_{i}^{(p)}\right) \\
m_{i}^{(l)}+\mu_{i}^{(l)}
\end{array}\right) \\
& \times \prod_{r=1}^{R}\left[P_{r}^{*}\right]^{T(r)}
\end{aligned}
$$

где сумма $\sum_{\mu_{i}^{(l)}}$ берется по неотрицательным значениям величин $\mu_{i}^{(l)}$, $i: J_{i} \neq \varnothing, l=1, \ldots, N_{i}$;

$$
P_{r}^{*}=\mathbf{P}\left\{s_{1, l}(i), \ldots, s_{m_{i}^{(l)}+\mu_{i}^{(l)}, l}(i) \in S_{t, r}\left(J_{i}^{(l)}\right) ; i: J_{i} \neq \varnothing, l=\overline{1, N_{i}}\right\}
$$

и в правой части (59) указан фиксированный набор индексов.

Обозначим $\tilde{v}=\tilde{v}(x)$ число координат вектора $x$ с индексами из множества

$$
\left\{s_{1, l}(i), \ldots, s_{m_{i}^{(l)}+\mu_{i}^{(l)}, l}(i) ; i: J_{i} \neq \varnothing, l=\overline{1, N_{i}}\right\}
$$

входящих в уравнение (30). Пусть

$$
\sum_{i, l}^{*}\left(m_{i}^{(l)}+\mu_{i}^{(l)}\right)=\sum_{i: J_{i} \neq \varnothing} \sum_{l=1}^{N_{i}}\left(m_{i}^{(l)}+\mu_{i}^{(l)}\right)=o(n)
$$


Разлагая вероятность (59) по формуле полной вероятности относительно системы событий $\tilde{v}=0, \tilde{v}=1, \tilde{v} \geqslant 2$, аналогично выкладкам (32) найдем

$$
\begin{aligned}
P_{r}^{*}= & {\left[\left(\begin{array}{c}
n \\
d(r)
\end{array}\right) \frac{d(r) !}{\left|\Sigma_{\varphi_{r} \mid}\right|}\right]^{-1} } \\
& \times\left[\left(\begin{array}{c}
n-\sum_{i, l}^{*}\left(m_{i}^{(l)}+\mu_{i}^{(l)}\right) \\
d(r)
\end{array}\right) \frac{d(r) !}{\mid \Sigma_{\varphi_{r} \mid}}\right. \\
& +\sum_{i, l}^{*}\left(m_{i}^{(l)}+\mu_{i}^{(l)}\right) \sum_{\substack{d_{0}, \ldots, d_{q-1} \\
d_{0}+\cdots+d_{q-1}=d(r)-1}} \prod_{\substack{J_{i} \neq \varnothing \\
\text { (l) }}}\left(\begin{array}{c}
n_{i}-\sum_{l}\left(m_{i}^{(l)}+\mu_{i}^{(l)}\right) \\
d_{i}
\end{array}\right) \\
& \times \prod_{i: J_{i}=\varnothing}\left(\begin{array}{c}
n_{i} \\
d_{i}
\end{array}\right) \pi_{r}\left(J_{i}^{(l)} ; d_{0}, \ldots, d_{q-1}\right) \frac{\prod_{i=0}^{q-1} d_{i} !}{\left|\Sigma_{\varphi_{r}}\right|} \\
& \left.+O\left(n^{d-2}\left(\sum_{i, l}^{*}\left(m_{i}^{(l)}+\mu_{i}^{(l)}\right)\right)^{2}\right)\right],
\end{aligned}
$$

где $\pi_{r}\left(J_{1}^{(l)} ; d_{0}, \ldots, d_{q-1}\right)=\sum_{k=1}^{d(r)} \pi_{r}^{(k)}\left(J_{1}^{(l)} ; d_{0}, \ldots, d_{q-1}\right)$ и величина $\pi_{r}^{(k)}\left(J_{1}^{(l)} ; d_{0}, \ldots, d_{q-1}\right)$ равна числу множеств вида

$$
\left\{\begin{array}{c}
\left(y_{1}, \ldots, y_{k}, \ldots, y_{d(r)}\right): y_{k} \in i \cup J_{i}^{(l)} ; \quad y_{\nu}=C_{\nu}, \quad \nu \neq k ; \\
\varphi_{r}(y)=C=\mathrm{const} \forall y_{k} \in i \bigcup J_{i}^{(l)}
\end{array}\right\},
$$

соответствующих различным фиксациям $y_{\nu}=C_{\nu}$ переменных $y_{\nu}, \nu \neq k$, таким, что $d_{w}$ переменных $y_{\nu}, \nu \neq k$, принимают значение $w=$ $0, \ldots, q-1$.

Выражение (60) перепишем в следующем виде:

$$
\begin{aligned}
P_{r}^{*}= & -\frac{1}{n} \sum_{i, l}^{*}\left(m_{i}^{(l)}+\mu_{i}^{(l)}\right)\left(d(r)-\tilde{\lambda}_{r}\left(J_{i}^{(l)}\right)\right) \\
& +O\left(\left(\frac{1}{n} \sum_{i, l}^{*}\left(m_{i}^{(l)}+\mu_{i}^{(l)}\right)\right)^{2}\right),
\end{aligned}
$$

где

$$
\tilde{\lambda}_{r}\left(J_{i}^{(l)}\right)=\sum_{\substack{d_{0}, \ldots, d_{q-1} \\ d_{0}+\cdots+d_{q-1}=d(r)-1}}\left(\theta_{0}(n)\right)^{d_{0}} \cdots\left(\theta_{q-1}(n)\right)^{d_{q-1}} \pi_{r}\left(J_{1}^{(l)} ; d_{0}, \ldots, d_{q-1}\right) .
$$

Из определения величины $\pi_{r}^{(k)}\left(i, j ; d_{0}, \ldots, d_{q-1}\right)$ (см. (5) и ниже) вытекает $\pi_{r}^{(k)}\left(J_{i}^{(l)} ; d_{0}, \ldots, d_{q-1}\right)=\pi_{r}^{(k)}\left(i, j ; d_{0}, \ldots, d_{q-1}\right), \forall j \in J_{i}^{(l)}$. Следовательно,

$$
\tilde{\lambda}_{r}\left(J_{i}^{(l)}\right)=\lambda_{r}(i, j)+o\left(\ln ^{-1} n\right), \quad \forall j \in J_{i}^{(l)},
$$




$$
\sum_{r=1}^{R} t(r) \widetilde{\lambda}_{r}\left(J_{i}^{(l)}\right)=\lambda^{*}+o\left(\ln ^{-1} n\right)
$$

Поэтому при условиях $3.2,3.3$ теоремы 1 и при условии $\mu_{i}^{(l)} \leqslant \ln n$, используя (61), найдем

$$
\begin{aligned}
\prod_{r=1}^{R}\left[P_{r}^{*}\right]^{T(r)}= & n^{-\sum_{i, l}{ }^{*}\left(m_{i}^{(l)}+\mu_{i}^{(l)}\right)} \\
& \times \exp \left\{-(z+o(1)) \sum_{i, l}^{*}\left(m_{i}^{(l)}+\mu_{i}^{(l)}\right)+O\left(\frac{(\ln n)^{3}}{n}\right)\right\} .
\end{aligned}
$$

Из (58), (62), с учетом неравенств Бонферрони для формулы включения и исключения [5], получим искомую оценку

$$
\mathbf{P}\left\{\left|S_{i}^{(l)}\right|=m_{i}^{(l)}\right\}=\prod_{i: J_{i} \neq \varnothing} \prod_{l=1}^{N_{i}} \frac{\left(\theta_{i} e^{-z}\right)^{m_{i}^{(l)}}}{m_{i}^{(l)} !} \exp \left\{-\theta_{i} e^{-z}+o(1)\right\} .
$$

Оценим теперь вероятность $\mathbf{P}\{\eta>0\}$. Зафиксируем некоторую пару переменных

$$
x_{s_{1}\left(i_{1}\right)}, \quad x_{s_{2}\left(i_{2}\right)}
$$

где $s_{1}\left(i_{1}\right) \in\left\{s: x_{s}^{\circ}=i_{1}\right\}, s_{2}\left(i_{2}\right) \in\left\{s: x_{s}^{\circ}=i_{2}\right\}, i_{1}, i_{2}: J_{i_{1}}, J_{i_{2}} \neq \varnothing$, и где мы полагаем $i_{1} \leqslant i_{2}, s_{1}(i)<s_{2}(i)$ в случае $i_{1}=i_{2}=i$. Введем индикаторы

$$
I_{t, r}^{(k)}=I\left(s_{1}\left(i_{1}\right) \in S_{t, r}\left(J_{i_{1}}^{\left(l_{1}\right)}\right), s_{2}\left(i_{2}\right) \in S_{t, r}\left(J_{i_{2}}^{\left(l_{2}\right)}\right), C_{t, r}^{(k)}\right), \quad k=1,2,
$$

при некоторых фиксированных $l_{1}, l_{2}$. Каждый из этих индикаторов равняется единице, если выполняются все три события, указанные в скобках, и равняется нулю в остальных случаях. Событие $C_{t, r}^{(1)}$ выполняется, если не более одного переменного из пары (64) содержится в $(t, r)$-уравнении (в уравнении с номером $t$ из подсистемы уравнений с функцией $\varphi_{r}$ ). Событие $C_{t, r}^{(2)}$ выполняется, если оба переменных $(64)$ содержатся в $(t, r)$ уравнении.

Введем также индикатор

$$
I=I\left(s\left(i_{1}\right) \in S_{i_{1}}^{\left(l_{1}\right)}, s\left(i_{2}\right) \in S_{i_{2}}^{\left(l_{2}\right)}, C\right),
$$

где, согласно принятым ранее обозначениям, $S_{i}^{(l)}=\cap_{r=1}^{R} \cap_{t=1}^{T(r)} S_{t, r}\left(J_{i}^{(l)}\right)$; событие $C$ выполняется, если оба переменных (64) содержатся, по крайней мере, в одном уравнении системы.

С учетом (59), (61) имеем

$$
\begin{aligned}
\sum_{k=1}^{2} \mathbf{P}\left\{I_{t, r}^{(k)}=1\right\} & =\mathbf{P}\left\{s_{1}\left(i_{1}\right) \in S_{t, r}\left(J_{i_{1}}^{\left(l_{1}\right)}\right), s_{2}\left(i_{2}\right) \in S_{t, r}\left(J_{i_{2}}^{\left(l_{2}\right)}\right)\right\} \\
& =1-\frac{1}{n}\left(2 d(r)-\tilde{\lambda}_{r}\left(J_{i_{1}}^{\left(l_{1}\right)}\right)-\tilde{\lambda}_{r}\left(J_{i_{2}}^{\left(l_{2}\right)}\right)\right)+O\left(n^{-2}\right),
\end{aligned}
$$


причем $\mathbf{P}\left\{I_{t, r}^{(2)}=1\right\}=O\left(n^{-2}\right)$. Поэтому

$$
\mathbf{P}\{I=1\}=\prod_{r=1}^{R}\left[\sum_{k=1}^{2} \mathbf{P}\left\{I_{t, r}^{(k)}=1\right\}\right]^{T(r)}-\prod_{r=1}^{R}\left[\mathbf{P}\left\{I_{t, r}^{(1)}=1\right\}\right]^{T(r)}=O\left(\frac{\ln n}{n^{3}}\right)
$$

Следовательно, для вероятности $\mathbf{P}\{\eta>0\}$ с учетом равенства

$$
\eta=\sum I=\sum_{i_{1}, i_{2}} \sum_{s_{1}\left(i_{1}\right), s_{2}\left(i_{2}\right)} \sum_{l_{1}, l_{2}} I\left(s_{1}\left(i_{1}\right) \in S_{i_{1}}^{\left(l_{1}\right)}, s_{2}\left(i_{2}\right) \in S_{i_{2}}^{\left(l_{2}\right)}, C\right)
$$

и неравенств $i, l \leqslant q, s(i) \leqslant n$ выполняется оценка

$$
\mathbf{P}\{\eta>0\} \leqslant \sum \mathbf{P}\{I=1\}=O\left(n^{-1} \ln n\right) .
$$

В силу (63), (65) выполняется (56). Теорема 1 доказана.

3.2. Д ок а з т е л с т в т е о р м ы 2 существенно проще, так как вместо величин $\left|S_{i}^{(l)}\right|$ достаточно исследовать одну величину $|S|$, равную числу переменных вектора $\boldsymbol{x}$, не вошедших ни в одно уравнение системы (4). В эквивалентной терминологии эта величина равна числу пустых ячеек в схеме размещения частиц комплектами и при условии 3.2 теоремы 2 асимптотически распределена по закону Пуассона с параметром $e^{-z}$ (см. [3]). Ясно, что $\mathbf{P}\left\{\xi \geqslant q^{|S|}\right\}=1$. Из этого соотношения, пуассоновости величины $|S|$ и (24) следует (15) и (16).

Учитывая, что для обоснования пуассоновости величины $|S|$ и оценки (24) условие 3.1 не требуется, получаем последнее утверждение теоремы 2. Теорема 2 доказана.

3.3. Д оказате льст во те о р мы 3 покрывается доказательствами теорем 1,2 , если вместо лемм 1,2 использовать лемму 3.

\section{СПИСОК ЛИТЕРАТУРЫ}

1. Балакин Г.В. Графы систем двучленных уравнений с булевыми неизвестными. - Теория вероятн. и ее примен., 1995, т. 40, в. 2, с. 241-259.

2. Копытчев $B$. A. О распределении писла решений случайных заведомо совместных систем уравнений. - Теория вероятн. и ее примен., 1995, т. 40, в. 2, с. 430437.

3. Михайлов В.Г. Предельные теоремы для случайного покрытия конечного множества и для числа рештений системы случайных уравнений. - Теория вероятн. и ее примен., 1996, т. 41 , в. 2, с. 272-283.

4. Масол В. И. Теорема о предельном распределении числа ложных решенпй системы нелинейных случайных булевых уравнений. - Теория вероятн. и ее примен., 1998 , т. 43 , в. 1 , c. $41-56$.

5. Сачков В.Н. Комбинаторные методы дискретной математики. М.: Наука, 1977. 